\title{
Actualidad $^{*}$, indexicalidad ${ }^{* *}$ y estatus ontológico
}

\author{
( Juan Manuel Di Leo**** \\ BA-Logic, Lógica y Filosofía de la Lógica
}

\begin{abstract}
Resumen
El presente trabajo tiene como objetivo sostener una posición metafísica conocida como realismo modal que defiende la tesis según la cual el mundo actual y los mundos posibles tienen el mismo estatus ontológico, i.e. la tesis de la pluralidad de mundos (Lewis, 1986: 104). Para ello debo mostrar que la tesis de la indexicalidad de la actualidad puede sostenerse.
\end{abstract}

En este sentido dividiré el trabajo en cuatro apartados: en el primero haré una reconstrucción de los principales argumentos en favor de dicha tesis. En el segundo analizaré algunas objeciones hechas por Barrio (1999), centrándome en la afirmación según la cual el realismos modal no puede dar cuenta de la diferencia entre "existir" y "existir actualmente" sin caer en una argumentación circular o suponer lo que debe probar. En el tercero propondré tres contra argumentos a las objeciones de la sección anterior, el argumento modal, el ontológico y el epistémico. Y por último plantearemos las conclusiones.

\footnotetext{
Abstract

The present work aims to support a metaphysical position known as modal realism. This position defends the thesis according to which the actual world and possible worlds have the same ontological status, i.e. the thesis of the plurality of worlds. For this purpose, I must show that the thesis that supports the indexicality of actuality can be sustained.

In this regard we will divide the work into four sections: in the first one we will reconstruct the main arguments in favor of this thesis. In the second part we will analyze some objections made by Barrio, focusing on the assertion that modal realism cannot account for the difference between "existing" and "existing actuality" without falling into a circular argument or assuming what have to proved. In the third one, I

* El término en ingles "actual", "actuality", etc., serán traducidos por los términos en español "actual", "actualidad", más que por los términos "real", "realidad", etc. Sigo en esto a Barrio (1999).

** Uso la expresión "indexicalidad" y la familia de expresiones relacionadas con ella del mismo modo que Barrio (1999).

*** En 2018 el autor cambió su apellido a Gagino-Di Leo.
}

Keywords modal realism indexicality actuality 
will propose three counter-arguments to the objections of the previous section: the modal argument, the ontological argument, and the epistemic argument. And finally we will state the conclusions.

\section{Realismo y realismo modal}

Como dije al comienzo el realismo modal es una posición metafísica según la cual no hay una diferencia del estatus ontológico de los mundos posibles respecto del mundo actual. En este sentido, en el presente apartado nos dedicaremos a reconstruir los principales argumentos para sostenerla. Para ello tomaremos como guía la propuesta de David Lewis en Sobre la pluralidad de mundos (Lewis, 1986).

Antes será necesario destacar que tanto el actualismo como el realismo modal, se comprometen con la tesis según la cual hay dos dominios filosóficos, por un lado el dominio ontológico, y por otro lado el dominio del lenguaje. A su vez estos dos dominios están relacionados de modo tal que el dominio del lenguaje depende del dominio ontológico. El mundo es de un modo privilegiado e independiente del lenguaje, existe independientemente de que podamos enunciar algo sobre él. Como dice Lewis:

Nosotros hacemos lenguaje, conceptos, descripciones y representaciones imaginarias que se aplican a los mundos. Hacemos estipulaciones que seleccionan a algunos mundos y no a otros para traerlos a nuestra atención. Algunos de nosotros incluso hacemos afirmaciones al respecto de que otros mundos existen. Pero ninguna de estas cosas que hacemos son los mundos mismos (Lewis, 1986: 106).

\section{a. Presentación de la Tesis}

La forma de presentar su tesis de un modo intuitivo consiste en que si suponemos que la forma en la que las cosas son significa la manera en la que el mundo es y reconocemos que las cosas podrían haber sido de otras muchas maneras, entonces esas otras maneras en que las cosas podrían haber sido, son modos en que algún mundo es (Lewis, 1986: 105).

Ahora bien es importante destacar como dije al principio que la tesis del realismo modal equipara el estatus ontológico de los mundos posibles y el actual. En este sentido no reconoce la distinción hecha por las posiciones actualistas entre dos formas de existir: por un lado en sentido estricto, que se aplicaría al mundo actual, y por otro simpliciter, que se aplicaría al mundo posible (Lewis, 1986: 105).

Según la posición de Lewis un mundo es un gran objeto físico (Lewis, 1986: 103) (aunque podría contener entidades inmateriales), que no tiene límites espaciotemporales y esta causalmente cerrado. Toda manera en la que un mundo puede ser es una manera en la que algún mundo es. Los mundos no tienen relaciones espaciotemporales, no están a distancia o tiempo entre ellos. A su vez están causalmente aislados. Es por ello que los mundos no pueden ser creados desde fuera, sino que son plenamente existentes (Lewis, 1986: 103).

\section{b. Los Argumentos (la cuantificación y el paraíso)}

Hay dos argumentos centrales para sostener el realismo modal: por un lado el argumento de la cuantificación, y por otro lado el argumento del paraíso. Con respecto al primero podemos reconstruirlo como sigue: Si la cuantificación me compromete 
Por su parte el argumento del paraíso: Así como la teoría de conjuntos que postula ciertas entidades primitivas (conjuntos), permitió a los matemáticos unificar y economizar los compromisos y postulados teóricos, y a la vez los dotó de lo necesario para avanzar en sus esfuerzos. Del mismo modo la tesis de la pluralidad de mundos es para los filósofos el paraíso fértil para sus investigaciones. La idea del autor es que es aceptable comprometerse con una ontología controvertida de una teoría por mor de los beneficios teóricos que con ella se obtienen. Luego de lo cual el autor analiza cuatro aplicaciones de las modalidades con las que intenta mostrar cual es el alcance de los beneficios (Lewis, 1986: 106-108).

Ambos argumentos son independientes entre si. En este sentido un objetor debe poder mostrar que cada uno tiene problemas. Ahora bien para que la tesis se sustente el autor debe poder dar cuenta de la distinción entre el mundo actual y los mundos posibles. Según nuestras intuiciones a este respecto no parecen estar en pie de igualdad. Por ello la tesis de la pluralidad de mundos se completa con el análisis indéxical de la actualidad según la cual: "actual" refiere en el mundo $m$ al mundo $m$.

En este sentido "el mundo actual" debe entenderse como "este mundo". Ahora bien Lewis plantea que esta forma de entender la actualidad hace de ella una cuestión relativa. Un mundo es actual relativo a los habitantes de dicho mundo. "El aspecto relevante del contexto para el término "actual" es el mundo en el cual una determinada emisión tiene lugar" (Lewis, 1970: 184-185). Sin embargo esto no permite afirmar que "todos los mundos son actuales" es una oración verdadera, ya que no es cierto que "todos los mundos son este mundo".

Para finalizar podríamos reconstruir el argumento del paraíso de la siguiente forma:

1. Hay una relación entre el dominio metafísico y el dominio del lenguaje por la cual el segundo depende del primero.

2. Las semánticas modales son de gran utilidad teórica.

3. Para dar cuenta de las oraciones modales o bien se postula una distinción ontológica entre mundo actual y mundo posible, y se afirma que el segundo depende del primero, o bien no se postula tal diferencia y se explica la «actualidad» en términos de indexicalidad.

4. Si dos teorías explican lo mismo, debemos optar por aquella que es más simple.

5. Si se postula una diferencia ontológica entre mundos, entonces tenemos dos nociones de existencia y requerimos de dos explicaciones para dar cuenta de la verdad de las oraciones.

6. Si no se postula tal diferencia, entonces tenemos una única explicación que apela solo a una noción de existencia.

7. El realismo modal es más simple.

8. El realismo modal debe ser aceptado.

\section{Objeción de Barrio}

En el presente apartado me concentrare en revisar dos objeciones hechas por Barrio al planteo de Lewis sobre el realismo modal: La primera de ellas plantea que el análisis de la actualidad en dicha tesis requiere de un análisis relativistas, y por lo tanto no cumple con los propios criterios del autor para dar cuenta de nuestras intuiciones sobre aquello que consideramos actual. La segunda objeción es que si aceptamos las razones que Lewis sostiene para rechazar la posición alternativa, entonces esas mismas razones son suficientes para rechazar el realismo modal (Barrio, 1999: 50).

\section{a. Primera objeción}

Barrio parte de diferenciar dos problemas en la discusión sobre la semántica de la modalidad: por un lado un problema metafísico, relativo al estatus ontológico de 
los mundos posibles. Por otro lado un problema semántico o lógico relativo a como se caracteriza o que representación formal cumple mejor con nuestras intuiciones relacionadas con palabras como actualidad (Barrio, 1999: 49).

En cuanto al primer problema, como dije más arriba, la tesis de la pluralidad de mundos sostiene que no hay una distinción en cuanto al estatus ontológico entre el mundo actual y los mundos posibles. Los mundos posibles existen del mismo modo, en el mismo grado, que el mundo actual. Tomemos la reconstrucción de Barrio de la posición de Lewis.

\begin{abstract}
La argumentación básica que presenta Lewis en defensa de tal planteo comienza con una apelación a la intuición. El primer paso de tal estrategia es una consideración acerca del mundo en donde habitamos: el mundo en donde nosotros vivimos es algo completo. Tal completud es tanto espacial como temporal. Luego, continúa diciendo que, ante la pregunta sobre lo que es un mundo, responderemos seguramente que es el modo en que las cosas son, y que acaso, como él cree concluiremos que es un gran objeto físico. El paso siguiente resulta fundamental. Todos admitiremos que las cosas podrían haber sido de diferentes modos. Luego, si hay otros modos en que las cosas podrían haber sido, hay otros mundos posibles. Y nuestro mundo no es sino uno de muchos posibles. Con esta inferencia Lewis pretende justificar, aunque no en forma concluyente, el realismo modal (Barrio, 1999:51).
\end{abstract}

Entonces tenemos que:

1. El mundo en donde nosotros vivimos es algo completo espacio-temporalmente

2. Ante la pregunta por ¿Qué es el mundo? Respondemos: es el modo en que las cosas son.

3. El mundo es un gran objeto físico.

4. Todos admitimos que las cosas podrían haber sido de diferentes modos.

5. Si hay otros modos en que las cosas podrían haber sido, entonces hay otros mundos posibles.

6. Hay mundos posibles

Ahora bien, por un lado Barrio plantea que el paso central del que se debe dar cuenta en la posición de Lewis es el que va de 4 a 6 . Y por otro lado esta equiparación del estatus ontológico, hace necesaria una explicación de nuestras intuiciones respecto de la distinción entre el mundo actual y los mundos posibles, tal como advierten Barrio y Lewis.

En este sentido juega un papel crucial la explicación del segundo problema para dicha tesis. La presentación de la indexicalidad de la actualidad hecha por Barrio (1999: 54-55) es como sigue:

1. El mundo actual es el mundo en el que habitamos

2. En este sentido el análisis indexical del término actual, funciona como un indexical puro en términos de Kaplan, ya que es afectado sistemáticamente por las condiciones en que se emite. Sin embargo una vez fijadas las condiciones de emisión la referencia de "el mundo actual" no cambia.
2. "el mundo actual" significa "este mundo" 2

3. No tiene sentido hablar de la verdad de la oración tipo "Actualmente existo".

4. "Actualmente existo" es verdadera en cualquier ocasión posible de emisión en cualquier mundo posible.

5. "Este es el mundo actual" es verdadera siempre que sea emitida en cualquier mundo posible.

6. "Todos los mundos son actuales" es siempre falsa.

\section{b. Segunda objeción}

La segunda objeción de Barrio es planteada en los siguientes términos: en el otro extremo de la disputa con el realismo modal, las posiciones actualistas hacen colapsar 
lo actual y lo existente. En esta posición los mundos posibles no forman parte de lo existente, en el mismo sentido que el mundo actual. Siguiendo al autor (Barrio, 1999: 58) la posición de Lewis es que:

1. O bien los actualistas suponen lo que intentan probar (que el realismo modal es falso) o bien que confunde "existir..." con "existe actualmente"

2. Solo si los actualistas mostraran que solo lo actual es lo existente, entonces la tesis de la pluralidad de mundos seria falsa.

Por su parte Barrio afirma que según Lewis,

3. Si lo actual es relativo a los habitantes de un mundo, entonces la única diferencia que existe entre el mundo actual y los mundos posibles es la forma en que los habitantes de uno y otro se relacionan (Barrio, 1999: 59).

Ahora bien para poder dar cuenta de la relación entre los habitantes de un mismo mundo Lewis plantea dos criterios distintos, por un lado se debe cumplir con:

(i) Formar parte del mismo espacio-tiempo

(ii) Tener cadenas causales con nosotros.

Sin embargo, Barrio plantea que esta explicación es insatisfactoria, ya que no parece poder dar cuenta de la distinción entre entidades como Zeus o Pegaso y entidades como los números, que no cumplen ninguna de ellas con los dos criterios, pero que sin embargo nosotros estaríamos dispuestos a considerar a los últimos como parte de nuestro mundo. En este sentido Lewis propone un segundo criterio que apela a la noción de causalidad:

(i) Dos individuos son parte del mismo mundo si y solo si hay relaciones causales entre ellos.

En este sentido Lewis hace un análisis contrafáctico apelando a la noción de similaridad comparativa entre mundos por medio del cual pretende mostrar que los mundos son causalmente cerrados, luego de lo cual muestra la imposibilidad de la causación trasmundana y por ultimo concluye que dicha imposibilidad es sustento suficiente de la asimetría entre mundo actual y mundo posible (Barrio, 1999: 56-61).

Para finalizar podemos reconstruir los principales argumentos de la segunda objeción de Barrio al realismo modal de Lewis:

I. si bien este criterio le permite a Lewis mostrar que si dos eventos están causalmente conectados, ellos pertenecen al mismo mundo. No le permite mostrar que si dos eventos no pueden estar causalmente conectados, ellos pertenecen a distintos mundos, para explicar la asimetría entre mundo actual y mundo posible.

II. Tampoco es contundente el argumento del paraíso ya que considera que el hecho de que una teoría funcione explicativamente no es suficiente para la aceptación de las entidades de las que la teoría habla.

III. Aunque admita el criterio de utilidad, nada, en el formalismo de la definición de verdad para las oraciones modales, indica que debamos comprometernos con la idea de que los mundos posibles sean entidades concretas con las características que Lewis les atribuye. 
IV. Mostrar que varios problemas son similares y que las expresiones involucradas en cada uno de ellos comparten una característica semántica especial, no basta para mostrar que efectivamente esa característica explica el problema.

V. Lewis no puede dar cuenta de la distinción entre "existir" y "existir actualmente" ya que o bien recurre a una explicación circular por medio de la cual primero analiza los operadores modales como cuantificadores sobre mundo y después analiza la existencia por medio de la noción de posibilidad, o bien debe suponerse objetos en el dominio del cuantificador existencial que no existen actualmente, pero esto supondría lo que intenta ser probado.

\section{Contraargumentos}

En este apartado mostrare que la tesis de la indexicalidad de la actualidad puede ser sostenida sin apelar a la justificación circular entre cuantificadores modales y existenciales. Es decir mostrare que no es cierto el punto V planteado por Barrio. Cabe destacar considero que los argumentos I-IV, son independientes del argumento V.

Es decir que si bien los criterios para establecer las relaciones entre los habitantes de un mundo propuestos por Lewis pueden tener problemas con las entidades abstractas (números, conjuntos, etc) como afirma I, y el argumento del paraíso puede no ser un buen argumenta como afirma II, y aunque fuera un buen argumento eso no me compromete con que los mundos sean como los describe Lewis, por III, y que podemos estar en todo de acuerdo con IV. Eso no implica que deba hacerse una defensa circular para dar cuenta de la distinción entre mundo actual y mundo posible.

Mostrare que hay algo interesante en la diferencia entre "existir" y "existir actualmente" y que sostener que no hay tal diferencia nos compromete con restricciones problemáticas en los dominios de la cuantificación.

Tesis: a) "Necesariamente yo existo actualmente" no tiene las mismas condiciones de verdad que b) "necesariamente yo existo"

Antes de emprender dicha tarea me interesa marcar algunas aclaraciones preliminares: En primer lugar las oraciones que consideraré, están tomadas del lenguaje natural y por lo tanto pueden ser interpretadas de distintos modos. Particularmente me interesa marcar que dichas oraciones están sujetas a lo que se conoce como ambigüedad de alcance ya que poseen cuantificadores. En este sentido no pretendo afirmar que la interpretación que yo sigo sea la única posible, sino que desde esta interpretación del alcance de los cuantificadores, las oraciones (a) y (b) no tienen las mismas condiciones de verdad.

En segundo lugar debe tenerse en consideración la distinción entre oración tipo y oración caso. Una oración tipo no tiene valor de verdad, son las oraciones caso, donde se instancia la oración tipo la que puede ser evaluada.

En tercer lugar debe tenerse en mente la distinción entre contexto e índice planteada por Lewis (Lewis, 1980: 235-255). El autor afirma:

En los rasgos de contenido hay implicados dos tipos de dependencia de la verdad: la dependencia de los contextosy la dependencia de los índices. Un contexto es una localización (...) donde se pronuncia una oración. El contexto tiene un número incontable rasgos, determinados por el carácter de la localización. Un índice es una n-tupla de rasgos de contexto (Lewis, 1980: 235). 
Los índices pueden ser desplazados un rasgo a la vez, los contextos no.

\section{i. Argumento modal:}

Para comenzar me interesa marcar que la formulación del problema apelando al infinitivo parece favorecer la intuición de Barrio ya que dicha forma verbal comparte características propias de un sustantivo. En este sentido parece más pertinente analizar las dos oraciones formuladas en el presente del indicativo, ya que permite explicitar el tratamiento de la existencia como un modo de cuantificación:

a) Necesariamente yo existo actualmente.

b) Necesariamente yo existo.

Si existir colapsa con actual, entonces ambas oraciones tienen las mismas condiciones de verdad. Ahora bien podemos ver que:

1. (a) tiene las mismas condiciones de verdad que (b) Sup

2. Es perfectamente posible considerar un mundo alternativo al actual, idéntico en todo excepto en que en él que yo no existo. ${ }^{3}$

3. Si ese mundo es accesible desde el nuestro, entonces "Necesariamente yo existo" es falsa en este mundo.

4. Sin embargo "Necesariamente yo existo actualmente" no puede ser falsa en ningún mundo posible, enunciada por cualquier individuo de ese mundo. ${ }^{4}$ (Barrio)

5. Entonces, "Necesariamente yo existo actualmente" y "Necesariamente yo existo" no tiene las mismas condiciones de verdad.

\section{ii. Argumento ontológico:}

Más allá de lo que acabamos de mostrar, es importante tener en cuenta nuestras intuiciones con respecto a nuestra propia existencia, o la existencia de otras entidades. Es perfectamente aceptable que uno de los modos en que las cosas podrían haber sido, es tal que yo no hubiera existido. Estos tipos de mundos especialmente parecidos al nuestro son los más usados en semánticas modales para pensar contra-fácticos. En este tipo de argumentos se establecen clausulas ceteris paribus, por las cuales se supone que todo lo demás permanece constante. Es evidente que tenemos la pretensión que mi existencia, como la de cualquier otra entidad, no es necesaria en todos los mundos posibles, parece perfectamente posible que yo como cualquier otra entidad no exista en otro mundo diferente.

Ahora bien, podemos introducir un nuevo operador lógico @ (operador de actualidad $)^{5}$ cuya cláusula de valuación es:

$$
V(@ \phi)=1 \text { sii } V(\Phi)=1 \text { en el mundo actual }
$$

A su vez debemos considerar al indexico "yo" como:

a) $Y o=x$ tal que $x$ emite la oración $a=E x[a]$
3. Es decir desplazar el rasgo del índice del contexto relativo al mundo posible.

4. No puede desplazarse independientemente ningún rasgo del índice del contexto.

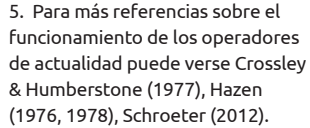

5. Para más referencias sobre el funcionamiento de los operadores de actualidad puede verse Crossley \& Humberstone (1977), Hazen $(1976,1978)$, Schroeter (2012)

6. Es discutible ¿cuál es el alcance del operador modal? Esto se conoce como ambigüedad de alcance de los cuantificadores. Uso está formalización de la oración del lenguaje natural entendiendo que es la más acorde con el significado corriente. Sin embargo esto no es central, ya que hay una oración del lenguaje natural tal que ella es formalízable con un operador de actualidad con el alcance indicado.
1. (a) tiene las mismas condiciones de verdad que (b)

2. "Necesariamente yo existo actualmente" puede formalizarse como: 6

a) $x(@(E x[a]))$

3. "Necesariamente yo existo" se formaliza como: b) x(Ex[b])

4. Ahora bien el operador modal en (a) hace que el cuantificador existencial ran- 
gueé sobre los de dominios todos y cada uno de los mundos posibles, pero el operador de actualidad, restringe dicho rango al mundo actual, rigidífica el dominio de la valuación. Entonces la verdad de (a) es evaluada solo en el dominio de mundo donde es emitida la oración.

5. Sin embargo el operador modal en (b) no está restringido por el operador de actualidad, y por ello la valuación de (b) tiene como rango todos los mundos posibles, y por el cuantificador existencial los dominios de cada uno de ellos.

6. Ahora bien, o bien (b) es falsa o bien asumo un compromiso ontológico de la necesidad de mi propia existencia a través de todos los mundos.

7. Si asumo ese compromiso ontológico, no hay razón para recortarlo en mi persona. En este sentido los dominios de los mundos posibles estarían determinados por el dominio del mundo actual.

8. Si los dominios de los mundos posibles están determinados por el dominio actual, entonces hay algún tipo de dependencia.

9. Si asumo dicha dependencia, entonces hay una diferencia ontológica entre el mundo actual y los mundos posibles

10.No puede asumir dicha dependencia.

11. (b) es falsa

12. (a) no tiene las mismas condiciones de verdad que (b)

Para finalizar es importante destacar que, parafraseando a Lewis, si los dominios de los mundos posibles están determinados, o dependen del mundo actual, entonces se supone una diferencia ontológica entre mundo actual y mundo posible, punto que el actualista debe probar. Por último es importante marcar que hay propuestas que suponen que los dominios de los mundos están fijos (Fara $\&$ Williamson, 2005), sin embargo este es un supuesto metafísico auxiliar e independiente, por lo tanto la carga de la prueba está de su parte.

\section{iii. Argumento Epistémico:}

Ahora bien hay un último argumento que reforzara lo dicho hasta acá. Llamémoslo el argumento del ignorante o argumento epistémico. La idea es la siguiente:

Supongamos que un sujeto se despierta un día en algún lugar, habiendo perdido todo conocimiento sobre cualquier cosa. No sabe nada de física o de metafísica. Desconoce que es él, cuál es su relación con las otras entidades del mundo, o tan siquiera si hay tal relación, incluso podría no saber que existen otras entidades. Su único conocimiento es la semántica de mundos posibles. Ahora bien, formula dos oraciones:

a) Necesariamente yo existo actualmente

b) Necesariamente yo existo

Luego de lo cual se pregunta por la verdad de las mismas. En dichas circunstancia sabe que (a) debe ser verdadera. Ahora bien, ¿puede saber que (b) es verdadera? Evidentemente no. No sabe nada de los dominios de los otros mundos posibles, no sabe nada de si hay o no dependencia metafísica entre los mundo o cual es esta dependencia. Pero entonces si requiere de un conocimiento que no tiene para saber que si es verdadera o falsa. En estas circunstancias cognitivas tan austeras entonces también sabe que (a) y (b) no son equivalentes.

\section{Conclusión}

Hemos mostrado que la tesis de la pluralidad de mundos descansa sobre dos argumentos independientes: por un lado el argumento de la cuantificación y por otro el 
argumento del paraíso. A su vez mostramos que dicha tesis requiere de un análisis indexical de la noción de actualidad, que permita dar cuenta de nuestras intuiciones respecto de la asimetría entre mundo actual y mundo posible. Por ultimo hemos demostrado que no es necesaria una justificación circular, como afirma Barrio, de la noción de actualidad presentando una explicación alternativa a través de tres argumento: i- el argumento modal, ii- el argumento ontológico, iii- el argumento epistémico. Estos muestran que al equiparar "existir" con "existir actualmente" tenemos tres problemas: en primer lugar si (a) y (b) tienen las mismas condiciones de verdad, entonces nuestras intuiciones sobre nuestra propia existencia fallan. En segundo lugar, que, o bien tomamos como supuesto que los dominios de los mundos posibles dependen de algún modo del mundo actual, y por ello suponemos lo que debemos probas, o bien suponemos que los dominios de los mundos posibles son extensionalmente idénticos al mundo actual y están fijos, pero dicha tesis metafísica es independiente y parece ser realmente controvertida. En tercer lugar, que hay una diferencia en el conocimiento que requerimos para afirmar la verdad de (a) y el que requerimos para afirmar la verdad de (b), y que con el conocimiento que es suficiente para afirmar la verdad de (a) no podemos afirmar la verdad ni la falsedad de (b). 


\section{Q Bibliografía}

" Barrio, E. A. (1999). "Indexicalidad y realismo modal", Cuadernos de Filosofía, $n^{\circ}$ $45,49-67$.

"Crossley, J. \& Humberstone, L. (1977). "The Logic of 'Actually'”, Reports on Mathematical Logic, $n^{\circ} 8,11-29$.

” Fara, M. \& Williamson, T. (2005). "Counterparts and actuality", Mind, $n^{\circ}$ 114(453), 1-30.

"Hazen, A. (1976). "Expressive Completeness in Modal Languages", Journal of Philosophical Logic, $n^{\circ}$ 5, 25-46.

" Hazen, A. (1978). "The Eliminability of the Actuality Operator in Propositional Modal Logic", Notre Dame Journal of Formal Logic, n 19, 617-622.

» Lewis, D. (1970). "Anselm and Actuality”, Nous 4, 175-188.

»Lewis, D. (2004 [1980]). “El índice, el contexto y el contenido”, en López Palma, H. (eds), La deixis, 235-255.

» Lewis, D. (2015 [1986]). Sobre la pluralidad de mundos, Tr. García Ramírez, E. México: UNAM.

" Kaplan, D. (2004 [1977]). “Los demostrativos”, en López Palma, H., La deixis. Lecturas sobre los demostrativos y los indíciales, Editorial Axas, 71-144.

» Kaplan, D. (2004 [1989]). Pensamientos Postreros, En López Palma H., La deixis. Lecturas sobre los demostrativos y los indíciales, Editorial Axas, 145-194.

"Schroeter, L. (2012). "Two-Dimensional Semantics", Stanford Encyclopedia of Philosophy, Recuperado de <https://plato.stanford.edu/entries/two-dimensionalsemantics/\#ModOpe>. 